\title{
A Novel Energy-Efficient Routing with Adaptive Receiver Using Cross-Layer Design in Wireless Sensor Networks
}

\author{
R. Abinaya ${ }^{1 *}$, C. Bhuvaneshwari ${ }^{2}$, R. Gayathri ${ }^{3}$ \\ Assistant Professor, Vel Tech Rangarajan Dr. Sangunthala R\&D institute of science and technology \\ *Corresponding author E-mail: abiabinaya12@gmail.com
}

\begin{abstract}
The increasing demand of wireless sensors in mission critical applications renders the Quality-of-Service (QoS) to be one of vital issue in wireless sensor network. It is necessary to deal with the underlying variance between energy efficiency and QoS, since sensor networks have different resource constraints. To manage these constraints such as unreliable links, limited power and other environmental factors in sensor networks, cross-layer approach is designed to ensure QoS. Recent routing protocols rely on the duty-cycle which causes sensor nodes to lose ample amount of energy by monitoring idle channels. In addition, opportunistic forwarding of packet leads to multiple receiver's receiving the same packet which induces redundancy. To address these issues, we propose a novel Opportunistic Routing with Adaptive REceiver (O-RARE) protocol as an alternative to duty cycle mechanism. The proposed work aims at reducing idle listening and redundancy by enabling low-power receiver at physical layer. Hence O-RARE is designed to ensure reliable and adaptive routing in our work through interactions among cross-layer.
\end{abstract}

Keywords: Cross-layer approach; Duty-cycle; Idle listening; Low-power receiver; Opportunistic routing; Redundancy elimination

\section{Introduction}

Wireless Sensor Networks (WSNs) can be defined as a selfconfigured and infrastructure-less wireless network that can cooperatively pass their data through the network to sink [5]. Sensors deployed in WSN are energy limited devices and therefore energy efficient communication techniques are the main requirements in these networks [2]. The challenges involved with routing protocols in these networks are the resources limitation of the network such as critical resource constraints of sensor nodes [11], unreliable link and rough environmental conditions [1]. The extent of energy consumed by sensor due to radio communication is ample compared to other components in the network [7].

The routing protocols in recent days rely on the duty-cycle mechanism that allows sensor nodes to wake up synchronously or asynchronously for forwarding the packet [3]. So the sensor nodes tend to listen to the idle channel during their wake-up period which causes significant energy consumption [9]. In addition, the microcontroller unit of sensor node also loses its energy by overhearing packets sent to the neighboring nodes [8].

Moreover opportunistic routing protocol makes use of the broadcast medium of wireless communications during data forwarding [6]. Thus forwarding a packet in broadcast manner can cause multiple receivers to receive the same packet which leads to duplication [4]. Cross-layer design is required to provide efficacy through interactions in Physical layer to network layer [10].

In O-RARE approach, information about the lower layer is disseminated to higher layers for improving the performance of the network. At the physical layer, the power consumed by sensor node is controlled using a novel mechanism which triggers main radio on reception of data packet. Microcontroller unit in a sensor node is in control of radio which consumes energy by overhearing message that is not addressed to it.
Secondly, MAC layer is responsible for deciding upon channel utilization and SNR where scheduling has to be performed for efficient routing. Then in the network layer, reliable routing mechanism is used to route the packets with minimal energy consumption.

The objective of the work is to minimize energy consumption of sensor nodes and eliminating duplicate forwarders by using novel mechanism, Opportunistic RARE.

The rest of the paper is organized as follows. Section 2 presents the proposed O-RARE protocol. In Section 3, the experimental setup and the simulation results are given. The obtained results are discussed in Section 4. The paper is concluded in Section 5 with focused future work.

\section{Proposed Methodology}

In this section, the design of Opportunistic Routing with Adaptive REceiver (O-RARE) is described to address the above design challenges. A cross-layer design is devised between the network and MAC layers in which the QoS requirements are imposed in the next hop by using adaptive receiver. It is determined based on the link quality and residual energy of the neighboring nodes as shown in Figure 1. MAC layer is in charge for allocating shared wireless channel and for scheduling the packets for reliable transmission. MAC layer also enforces the link level QoS parameters through the process of scheduling. Opportunistic routing decision is based upon the residual energy of the sensor's node in the network layer along with the link rate adaption using admission control. Each node maintains its forwarder set and the node forwards its packet to any of the nodes in the set by computing forwarder score estimation. 


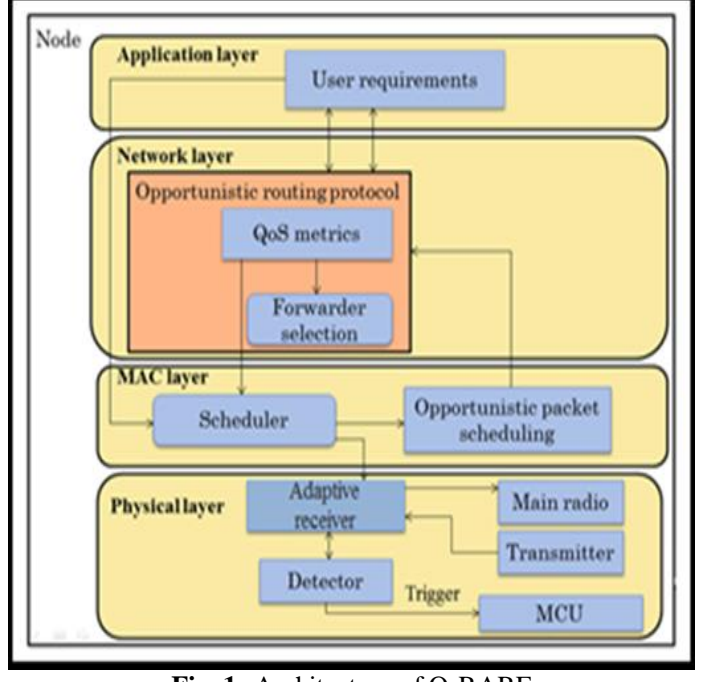

Fig. 1: Architecture of O-RARE

\subsection{Adaptive Receiver Mechanism}

The proposed novel mechanism given is the best candidate for replacing traditional duty cycled MAC protocols. Our QoS goal is to achieve QoS requirements in terms of energy consumption and reliability of data transmission while extending the network lifetime.

In scheduling phase, a methodology to provide a reliable delivery mechanism over an unreliable wireless medium is devised. The link quality is estimated initially for the set of forwarding candidates and then best relay node is selected as forwarder.

The solution focuses on tuning the receiver adaptively through limited flooding transmission for the receiving nodes. The packet is initially flooded to those neighboring candidates which satisfy required QoS constraints.

The chosen forwarder nodes will tune its power to low-power for receiving flooded packets. Once the packet is received by chosen forwarders, the best relay node among them will forward the packet similarly until it reaches destination. Thus through the limited flooding transmission and adaptive tuning of receiver at physical layer energy consumption is reduced.

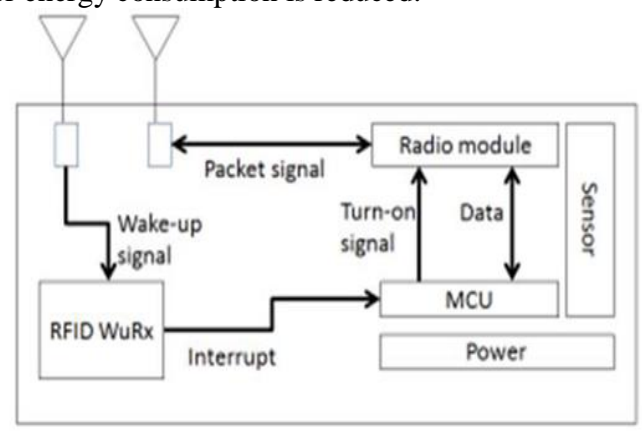

Fig. 2: Functioning of Adaptive receiver

To solve the problem of idle listening adaptive receiver is devised by enabling the nodes to wake up on demand using trigger mechanism. Node is controlled with low power mode until it receives packet addressed to it. So the scheduled duty cycle approach with periodic wake-up is replaced with this Opportunistic Routing with Adaptive REceiver mechanism. This proposed approach allows sensor nodes to switch from low power mode to optimal power mode on reception of trigger as shown in Figure 2.

\section{Simulation Setup}

All paragraphs must be justified alignment. With justified alignment, both sides of the paragraph are straight.
O-RARE protocol performance is simulated using OMNeT++, a Wireless Sensor Network simulator that supports the design of cross layer approach. We evaluated O-RARE with the former related routing methods such as CLAC, DOF, CL-HEEP in multihop wireless networks. The QoS parameters such as Energy, latency and throughput are considered as the performance metrics to do a fair comparison.

About 19 MICAz motes and Sink are evenly deployed and organized in the form of a matrix $4 \times 7$. B MAC protocol is used in the second layer that reflects realistic radio model that can vary the power of sensor nodes for packet transmission. To support mission critical applications in the realistic deployable environment critical parameters such as Beacon interval, Wake-up interrupt, Listen period, Wake-up signal etc are dynamically tuned in $\mathrm{O}$ RARE protocol.

Depending upon the size of network the parameters used for this experiment is given in Table 1 . Simulation is originated by sink node and by this means each node in the network creates its own look up table with the routing metrics. The power level is set up with the baseline power with general processing power.

Table 1: Experimental parameters

\begin{tabular}{|c|c|}
\hline Parameter & Value \\
\hline Sleep current & $570 \mathrm{nA}$ \\
\hline Receiver current & $24.5 \mu \mathrm{A}$ \\
\hline Bit rate & $4 \mathrm{kbps}$ \\
\hline Timeout window & $10 \mathrm{~ms}$ \\
\hline Interrupt call size & $35 \mathrm{bits}$ \\
\hline
\end{tabular}

The low-power in the node can preserve the energy for performing neighbor discovery and transmission purpose. In such situations 802.11 radio can save energy in the low power mode and can be turned on for critical situations.

Thereby the nodes can be coordinated for choosing best relay node and interrupting the relay node for packet transmission. For evaluating this, MiXiM framework is used in the OMNet++ supporting the extensions for estimating energy consumption. The transmission of data packets is initiated from a random node as per the requirements of application which generates packets to transmit. The packet is then forwarded with respect to the constraints of routing metrics opportunistically along with the adaptive receiver. The relay node has to be chosen dynamically by the forwarder node from the candidate set in this Opportunistic routing approach. Once the relay node is selected then the node then switches its power to optimal power mode and receives the packet.

\section{Results and Discussions}

The proposed O-RARE algorithm is evaluated under varied link quality and the experimental analysis of OMNET++ simulation results are conferred here. The link quality and reliability is ensured as proposed work opportunistically determines the path and tune the receiver to forward the packet.

Figure 3 depicts that energy consumption of Opportunistic Routing with Adaptive Receiver (O-RARE). Thus the obtained energy consumption seems to be less when compared to CL-HEEP, DOF and EEDS. The retransmission is avoided since algorithm operates based on the dynamic environment and overhearing can be eliminated due to the on-demand wake up.

Furthermore, the optimal power receiver selects the best forwarder node based on the constraints specified per hop path. The power consumption of the O-RARE is decreased and number of awaken forwarders is decreased as the duty cycle is decreased. The fluctuation in power consumption in the CLAC was because of duty cycle and its periodic wake up of sensor nodes. 


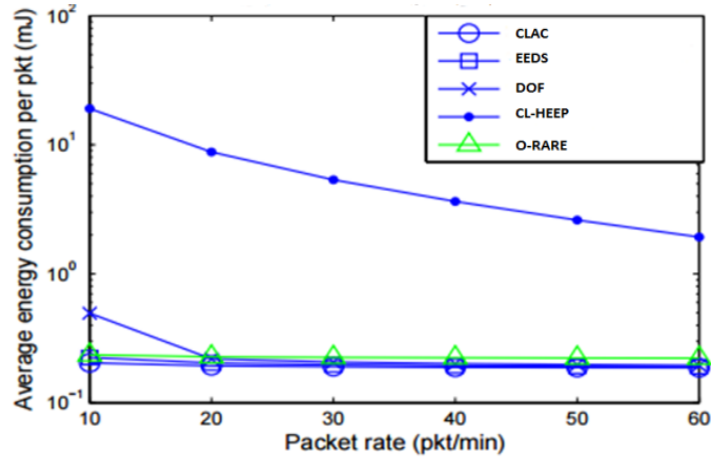

Fig. 3: Energy consumption

The CLAC chooses the first awake node as forwarder rather than best forwarder and retransmission count expires for awaken forwarders. Since O-RARE protocol uses selective broadcast for three best nodes overall power is slightly higher when compared to CLAC.

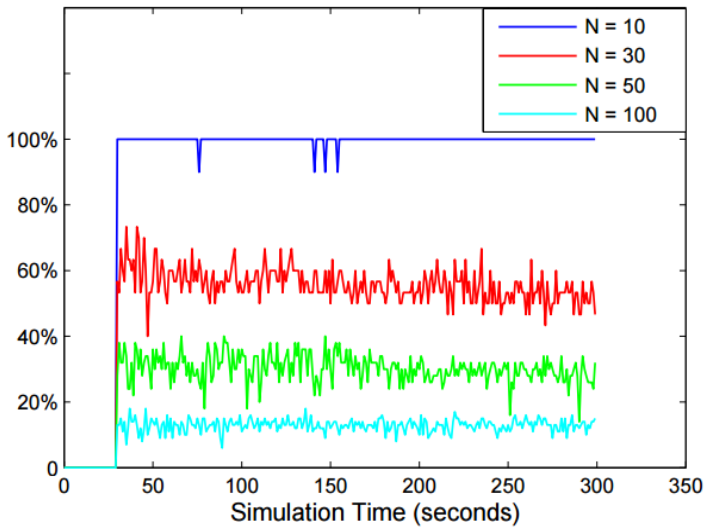

Fig.4: Delivery ratio

From the Figure 4, let $\mathrm{N}$ denote the number of nodes evaluated and the corresponding results depict delivery ratio. The nodes are prioritized as per the constraint of delivery ratio at different time period. O-RARE protocol achieves good delivery ratio for each forwarder without the need of retransmission.

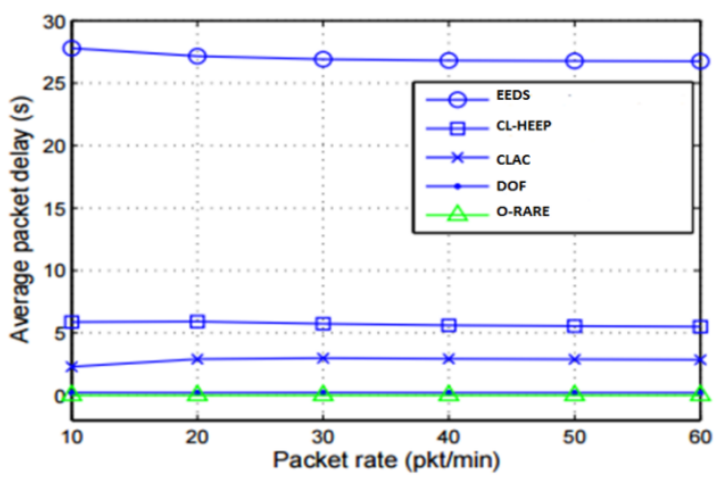

Fig. 5: Packet delay

Figure 5 shows the End-to-End Delay and a delivery ratio of ORARE, CL-HEEP and CLAC. From the Figure 5 it has been seen that CLAC attains $95 \%$ at 15 s delay constraint. ETX attains increased delivery rate over time and delivers $55 \%$ packet at $15 \mathrm{~s}$. CL-HEEP experienced lower delay than EEDS and attains $90 \%$ delivery ratio.

Thus O-RARE protocol outperforms the existing routing methods and achieves energy, reliability and better delivery ratio with user bound constraints.
The outcomes that gained from this work are listed as follows,

1. A cross-layer approach to guarantee the successful data transmission through Opportunistic Routing with Adaptive Receiver (O-RARE).

2. Designing low-power receiver to adaptively tune the power of sensor node for transmission to save the energy of sensor nodes.

3. Ensure QoS in terms of latency, reliability and energy in an effective way by considering joint functionalities of adaptive receiver and opportunistic routing across layers.

\section{Conclusion}

Cross layer plays a vital role in determining channel utilization and energy consumption for scheduling paths dynamically. Hence O-RARE is designed to eliminate redundancy and overhearing which might cause tremendous power loss in mission critical applications is controlled using interrupt call. Proposed work provides a good alternative for energy saving compared to the dutycycle approach and outperforms existing routing algorithms. The joint functionalities of reliable routing along with the low power receiver will reduce the energy consumption. In future the efficient and reliable routing mechanism will be implemented along with the security features for ensuring confidentiality of interrupt request since false alarms might cause energy consumption.

\section{References}

[1] Aliaa A. Youssif, Mohamed EzzElDienAbd El Kader, AtefZakiGhalwash(2016). Energy Aware and Adaptive Cross-Layer Scheme for Video Transmission Over Wireless Sensor Networks. IEEE Sensors Journal, 16(21), 7792-7802.

[2] Amir Darehshoorzadeh, AzzedineBoukerche(2015). Underwater sensor networks: a new challenge for opportunistic routing protocols. IEEE Communications, 53(11), 98-107.

[3] AmitSarkar, T. SenthilMurugan(2016). Routing protocols for wireless sensor networks: What the literature says? Elsevier.

[4] Azeddine, Bilami Sabrina Boubiche, FaouziHidoussi(2015). A Cross-Layer Communication Protocol with Transmission Power Adjustment for Energy Saving in Multi-hop MhWSNs. Springer, 85(1), 151-177.

[5] Daibo Liu, Xiaopei Wu, Zhichao Cao, Mingyan Liu, Yujun Li, MengshuHou(2015). CD-MAC: A contention detectable MAC for low duty-cycled wireless sensor networks. Sensing, Communication, and Networking conference.

[6] Fatima Z. Djiroun; DjamelDjenouri(2016). MAC Protocols with Wake-up Radio for Wireless Sensor Networks: A Review. IEEE Early Access Articles, 99, 1 - 1.

[7] Joaquim Oller, Ilker Demirkol, Jordi Casa demont, Josep Paradells, Gerd Ulrich(2016). Has Time Come to Switch From DutyCycled MAC Protocols to Wake-Up Radio for Wireless Sensor Networks? IEEE/ACM Transactions on Networking, 24(2).

[8] Jungmin So, Heejung Byun(2016). Load-Balanced Opportunistic Routing for Duty-Cycled Wireless Sensor Networks. IEEE Transactions on Mobile Computing.

[9] Michele Magno, TommasoPolonelli, Luca Benini, Emanuel Popovici(2015). A Low Cost, Highly Scalable Wireless Sensor Network Solution to Achieve Smart LED Light Control for Green Buildings. IEEE Sensors Journal.

[10] Naeimeh Omidvar, Mohammad Reza Pakravan, Danny H. K. Tsang(2015). Efficient Energy-Aware Routing With Redundancy Elimination. IEEE Journal On Selected Areas in Communications, 33(12), 2815-2825.

[11] Daibo Liu, Zhichao Cao, Jiliang Wang, Yuan He, MengshuHou, Yunhao Liu(2016). DOF: Duplicate Detectable Opportunistic Forwarding in duty-cycled wireless sensor networks. IEEE/ACM Transactions on Networking, 24(2), 662-673.

[12] Nessrine Chakchouk(2015). A Survey on Opportunistic Routing in Wireless Communication Networks. IEEE Communications Surveys \& Tutorials, 17(4), $2214-2241$. 